\title{
Nanotherapeutics to Overcome Conventional Cancer Chemotherapy Limitations
}

\author{
C. Moorthi*1, R. Manavalan ${ }^{1}$, K. Kathiresan ${ }^{1}$ \\ ${ }^{1}$ Department of Pharmacy, Annamalai University, Annamalai Nagar, Chidambaram, Tamil Nadu, India \\ Received, September 17, 2010; Revised, September 22, 2010; Accepted, February 3, 2011; Published, February 14, 2011.
}

\begin{abstract}
Cancer is one of the major causes of death worldwide and chemotherapy is a major therapeutic approach for the treatment which may be used alone or combined with other forms of therapy. However, conventional chemotherapy suffers lack of aqueous solubility, lack of selectivity and multidrug resistance. Nanotherapeutics is rapidly progressing aimed to solve several limitations of conventional drug delivery systems. Nonspecific target of cancer chemotherapy leads to damage rapidly proliferating normal cells and can be significantly reduced through folate and transferrin mediated nanotherapeutics which are aimed to target cancerous cells. Multidrug resistance is challenge in cancer chemotherapy which can be significantly reversed by solid lipid nanoparticles, polymeric nanoparticles, mesoporous silica nanoparticles, nanoparticulated chemosensitizer, nanoparticluated poloxamer and magnetic nanoparticles. Hydrophobic nature of chemotherapeutics leads to poor aqueous solubility and low bioavailability which can be overcome by nanocrystals, albumin based nanoparticles, liposomal formulation, polymeric micelles, cyclodextrin and chitosan based nanoparticles. This review focuses the role of nanotherapeutics to overcome lack of selectivity, multidrug resistance and lack of aqueous solubility of conventional cancer chemotherapy.
\end{abstract}

\section{INTRODUCTION}

Cancer is one of the major causes of the death worldwide. In India, cancer prevalence was estimated around 2.5 million with a growth of $8,00,000$ new cancer cases and 5,50,000 cancer deaths every year. Cancer of oral cavity, lungs, oesophagus and stomach are common among Indian males whereas cancer of cervix and breast are common among Indian females $(1,2)$. In United States, one out of four deaths is due to cancer and it is seen in all age groups and in both sex. In 2010, about 1,529,560 new cancer cases and 569,490 cancer deaths are estimated in United States by Cancer Statistics Review. Death among children below 14 years in the United States is due to cancer, particularly acute lymphocytic leukaemia followed by brain cancer and nonHodgkin lymphoma $(3,4)$. The three main approaches in cancer treatment are (a) Surgical excision, (b) Irradiation and (c) Chemotherapy. Comparative value of these approaches depends on tumor type and development stage of cancer. Major therapeutic approach for the treatment of localized and metastasized cancer is chemotherapy, which are used alone or combination with other forms of therapy $(5,6)$. However, conventional chemotherapy suffers some limitations (a) Limited aqueous solubility: Most chemotherapeutics either from plant source or synthetic are hydrophobic and requires solvents to formulate the dosage form which contribute to severe toxicity, (b) Lack of selectivity of anticancer drugs: Most chemotherapeutics lack selectivity toward cancerous cells cause significant damage to rapidly proliferating normal cells and (c) Multidrug resistance (MDR): MDR is mainly due to increased efflux pumps such as P-glycoprotein (Pgp) in the cell membrane which are responsible for transport of various anticancer drugs out of cells (7-10).

Nanotechnology literally means technology performed on a nanoscale. The nanoscale/ nanoparticles are ultrafine particles in the size of nanometre from $1 \mathrm{~nm}$ to $1000 \mathrm{~nm}$. Nanomedicine is an important area in nanotechnology which refers to highly specific medical intervention at the molecular scale for diagnosis, prevention and treatment of diseases (11). Nanotherapeutics are rapidly progressing field which are utilized to solve several limitations of conventional drug delivery system such as nonspecific biodistribution, lack of targeting, lack of aqueous solubility, poor oral bioavailability, and low therapeutic indices (12).

Some important technological advantages of nanotherapeutic drug delivery systems (NDDS) are (a) NDDS provides longer shelf life, (b) Both hydrophilic and hydrophobic substances can be incorporated in NDDS. (c) NDDS can be administrated through oral, nasal, parenteral, intraocular etc. (d) NDDS improve the biodistribution of cancer drugs.

Corresponding Author. C. Moorthi, Department of Pharmacy, Annamalai University, Annamalai Nagar, Chidambaram, Tamil Nadu, India. E-mail: cmoorthitgodu@gmail.com 
Whereas optimal size and surface characteristics of nanoparticles increases the circulation time of the drug, (e) NDDS provides control and sustain release of the drug both during the transportation and at the site of action and (f) NDDS increases the intercellular concentration of drug either by enhanced permeability and retention effect (EPR) or by endocytosis mechanism (13-14). This review focuses the role of nanotherapeutics to overcome lack of selectivity, multidrug resistance and lack of aqueous solubility of conventional cancer chemotherapy.

Nanoparticle drug delivery system to overcome lack of selectivity of anticancer drugs

Most cancer chemotherapeutics are administered either orally or intravenously to achieve systemic distribution for effective treatment. However, due to lack of selectivity these drugs cause significant damage to rapidly proliferating normal cells. The major goal of targeted therapies is to target the chemotherapeutics to cancer cell which ultimately reduce the side effects. Nanoparticles are targeted either passively or actively to specific sites.

\section{Passive targeting}

Size of nanoparticles and behaviour of tumor tissue vasculature plays a significant role in passive targeting (15). Solid tumor consists of tumor parenchyma and stroma, which inturn consist of vasculature and other supporting cells. Due to increased metabolic requirements of growing tumor cells, pre-existing blood vessels are subjected to angiogenic pressure and leads to the development of new capillaries to the tumor in a process called angiogenesis (16). Scanning electron microscopic studies revealed that the formed tumor capillaries are highly irregular and showed gross architectural changes. Normal tissue vasculatures are lined by tight endothelial cells thereby preventing entry of nanoparticle whereas tumor tissue vasculatures are leaky (gaps as large as 200 to $2000 \mathrm{~nm}$ between adjacent endothelial cells) and hyper permeable. This defective vascular architecture induces an EPR and permits accumulation of nanoparticles in the tumor interstitial space (Figure 1) (17-23).

Accumulation of nanoparticles in tumor tissues depends on interstitial fluid pressure which is higher in tumor tissues than in benign tumor and normal tissues. In particular, interstitial pressure would be higher at the centre diminishing towards the periphery which is responsible for induction of drugs outflow from the cells which may leads to drug redistribution in some portions of the cancer tissue. Accumulation of nanoparticles in tumor tissues are also depends on size, surface character, circulation half-life of the nanoparticles and the degree of angiogenesis of the tumor (24-26). Selected delivery systems to achieve passive targeting are liposomes, polymeric nanoparticles, nanocrystals, inorganic nanoparticles, micelles, and dendrimers etc.

\section{Active Targeting}

Paul Ehrlich coined the term "magic bullet" which is an idealized package that would target and deliver drugs to a specific place in the body and this idea of active targeting was proposed even before a rational targeting ligand was discovered (27). Active targeting involves conjugation of targeting molecules (like antibodies, ligands, peptides, nucleic acids etc.) on the surface of nanoparticles with receptors over expressed on a tumor cell surface. Tumor targeting molecules on the nanoparticles bind to cell through an endosome-dependent mechanism (Figure 2) which bypasses the drug efflux pump leading to high intracellular concentration $(23,28$ 30).

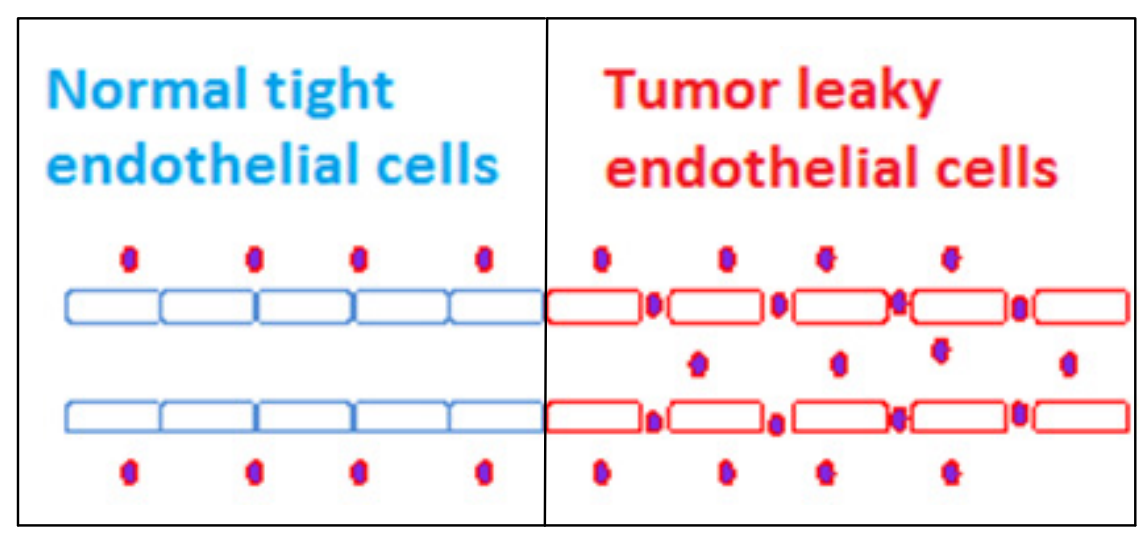

Figure 1. Nanoparticle accumulation in tumor tissue through EPR effect 


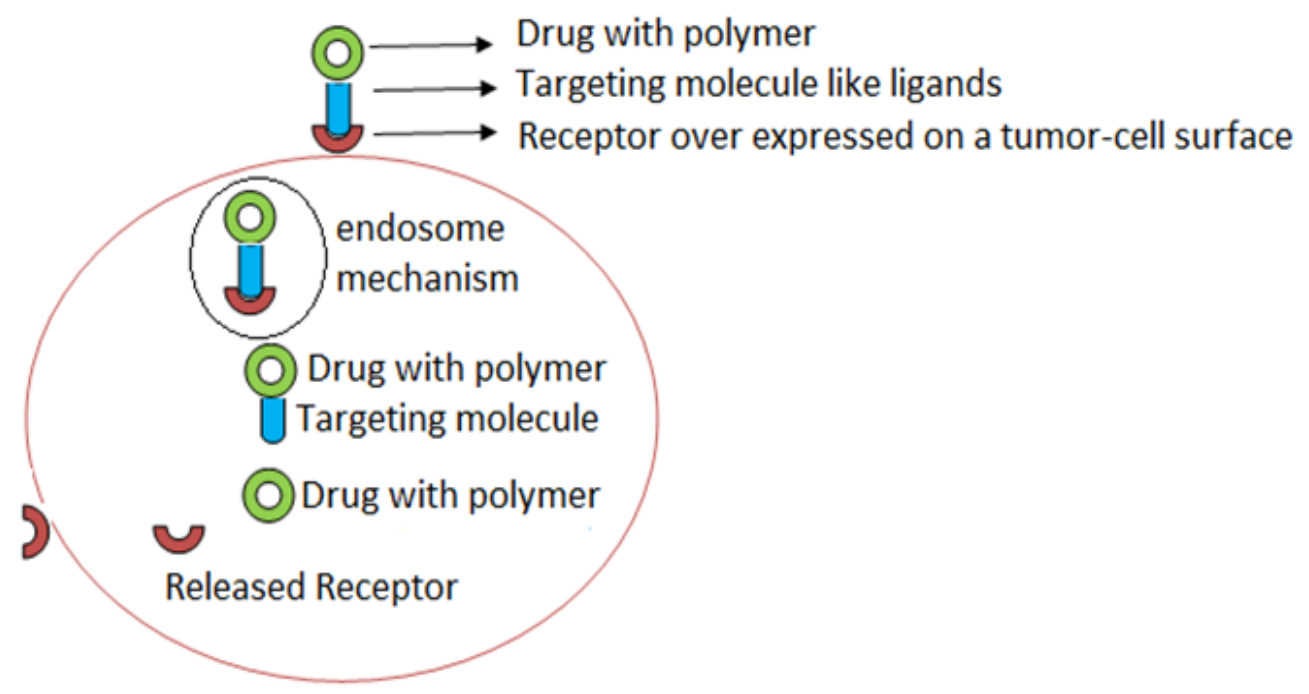

Figure 2. Endosome dependent mechanism of nanoparticles

\section{Folate-Mediated Targeting}

Folate receptor, a cell membrane associated glycosylphosphatidylinositol anchored glycoproteins involved in human growth and development, cell division and DNA synthesis, has been explored to target therapeutics into cancer cell due its over expression on malignant cancer cells. Binding of folic acid to folate receptor (FR- $\alpha$ and FR- $\beta$ ) initiates receptormediated endocytosis and internalization of folic acid. Most human tissues lack the folate receptor, except the placenta, choroids plexus, lungs, and kidneys; however, cellular activation and proliferation leads to over expression of highaffinity folate receptors in many cancers. Thus, Folate-mediated targeting has been used to deliver protein toxins, low-molecular weight chemotherapeutic agents, radio-imaging agents, MRI contrast agents, radio therapeutic agents, liposomes containing chemotherapeutic drugs, genes, antisense oligonucleotides, ribozymes, and immunotherapeutic agents to cancer cells (31-34). Many studies have been carried out to prove the enhancement of anticancer activity via folate mediated targeting.

To be specific, Zhaowu Zhang et al. utilized folate-conjugated nanoparticles on human cervices carcinoma cells and found no cellular uptake of folate-conjugated nanoparticles in A549 cells which lacks folate receptor (35). Similarly Fabiana Canal et al. proved that antiproliferative activity of epirubicin markedly increased going from Folate Receptor (-) to Folate Receptor (++) cells with greater cellular internalization with the folate targeted conjugates than with their nontargeted analogues which was confirmed by confocal microscopy studies (36). Chan Zhang et al. demonstrated uptake of folic acid conjugated doxorubicin up by HeLa cells and showed greater cytotoxicity compared to non-folate-mediated nanoparticles (37). Gene therapy for the treatment of cancer is a promising approach. However, clinical application of cancer gene therapy lacks vector that are safe, efficacious, and tumorselective. Targeted gene delivery through cellular receptors, using either viral or nonviral vectors, is emerging as a novel approach. Folate receptor targeted liposomes have been evaluated for the targeted delivery of antisense oligodeoxyribonucleotides (ODNs) and shown impressive folate receptor selectivity in cell culture assays and shown promising tumorspecific gene transfer activity in several in vivo models (38).

\section{Transferrin-Mediated Targeting:}

One of the characterized ligands to be exploited for targeting tumor cells is transferrin which plays an essential role in iron homeostasis and cell growth. Inherent characteristic of some cancer cells is over expression of transferrin receptor. However, high expression of transferrin receptor is seen hypothalamus and medulla oblongata compared to other part of brain and many in vivo studies showed that transferrin increases brain delivery of nanoparticles (39). Uptake of transferrin into cells is mediated by transferrin receptors which are cell membrane associated glycoprotein. Binding of transferrin to transferrin receptor initiates receptor mediated endocytosis and interlization of transferrin. Whereas in presence of inhibitors, transferrin mediated 
nanoparticles interact with the cells in a specific manner and enter the cells via the caveolae pathway $(40,41)$. Many studies have been carried out to prove the enhancement of anticancer activity via transferrin mediated targeting.

To be specific, $\mathrm{Yu}$ Zheng et al. studied the activity of transferrin-conjugated lipid-coated poly (d,l-lactide-co-glycolide) (PLGA) nanoparticles carrying the aromatase inhibitor, 7 $\alpha$-(4'-amino)phenylthio-1,4-androstadiene-3,17dione on breast cancer cells and results showed that aromatase inhibition activity of the transferrin nanoparticles was enhanced relative to that of the non-targeted nanoparticles, which was attributable to transferrin receptor mediated uptake (42). Similarly, Rohit S. Mulik et al. studied the anticancer activity of transferrin conjugated solid lipid nanoparticles of curcumin on MCF-7 breast cancer cells and results showed that the cell uptake and cytotoxicity increased considerably with transferrin conjugated solid lipid nanoparticles compared to curcumin solution. Transferrin conjugated nanoparticles enhance the antitumor activity via active target mechanism and also contributes to the photo stability and sustain release of drug (43).

\section{Nanoparticle drug delivery system to overcome multidrug resistance}

A major problem in cancer chemotherapy is multidrug resistance. Cancers such as non-small cancer, lung cancer, and rectal cancer may not respond to standard chemotherapy from the beginning which is called primary resistance or natural resistance. Whereas some sensitive tumors respond well to chemotherapy drugs in the beginning but develop acquired resistance later. The cell membrane, cytoplasm, and nuclear protein participate in resistance mechanisms. The mechanisms with known clinical significance are: a) activation of transmembrane proteins effluxing different chemical substances from the cells; b) activation of the enzymes of the glutathione detoxification system; c) alterations of the genes and the proteins involved into the control of apoptosis (especially p53 and Bcl-2). However, multidrug resistance is mostly due to increased efflux pumps in the cell membrane. The most common efflux pump in the cell membrane is Pglycoprotein (Pgp) and it transports various anticancer out of cells by using ATP.

Pgp is one of the membrane transporter superfamily having the ATP-binding cassette (ABC) with well-preserved homology of the site where ATP binds. Other efflux pumps of the mammalian cell membrane in $\mathrm{ABC}$ superfamily include multidrug resistance-associated proteins
(MRP) and breast cancer resistance proteins (BCRP). Acidic organelles in multidrug resistance cells are also contribute to developing resistance to chemotherapeutic drugs. Since most anticancer drugs are in an ionisable form, the $\mathrm{pH}$ of extracellular matrix and intracellular compartments are critical factors in determining drug partitioning and distribution. In some instance higher dose may be required to overcome multidrug resistance which in return, may enhance the toxicity of the treatment. To decrease the toxicity and to enhance the selectivity of existing drugs, many drug delivery systems have been developed in recent years (44-52). Various nanoparticluate drug delivery systems to overcome multidrug resistance are highlighted in this review.

\section{Chemosensitizers through NDDS}

Drugs such as verapamil and immunosuppressant Cyclosporine A would inhibit or reverse resistance by functioning as competitive substrates of Pgp and are called chemosensitizers. Clinical studies also showed that these drugs could reverse resistance to anticancer drugs. Xiang Rong Song et al. studied the effect of chemosensitizer (verapamil) using PLGA vincristine nanoparticles and found the following (a) administration sequence of vincristine and verapamil was significant for maximal therapeutic efficacy, (b) Highest reversal could be achieved when vincristine and verapamil administration simultaneously, (c) PLGA nanoparticles showed moderate MDR reversal activity on MCF-7/ADR cells, (d) Normal tissue drug toxicity and fewer drug-drug interactions are seen when vincristine and verapamil co-encapsulated (53).

\section{Mesoporous Silica Nanoparticles}

Mesoporous silica nanoparticles (MSNs) were first reported by Kuroda and co workers in 1990 in Japan and later developed by Mobil Corporation laboratories and named as Mobil Crystalline of Materials, or MCM-41. Silica nanoparticles with much larger (4.6 to 30) nanometer pores were produced at the University of California and named Santa Barbara Amorphous type material, or SBA-15. Some of the advantages of MSNs are (a) Particle sizes of MSNs can be tuned from 50 to $300 \mathrm{~nm}$, (b) Stable and rigid frame of MSNs allows for resistance to $\mathrm{pH}$, mechanical stress, and degradation, (c) Pore diameters of MSNs can be tuned between 2 and $10 \mathrm{~nm}$ allowing for different drug loadings, (d) MSNs have a high surface area and large pore volume allowing high loadings of drugs and (e) The interior and exterior surfaces of MSNs can be 
selectively functionalized with different moieties on either surface (54-57).

Alex M. Chen et al. studied the activity doxorubicin and Bcl-2-targeted siRNA on multidrug resistant A2780/AD human ovarian cancer cells utilizing MSNs and results showed that by delivering Doxorubicin and Bcl-2 siRNA simultaneously into cancer cells, the Bcl-2 siRNA can effectively silence the Bcl-2 mRNA and significantly suppress the non pump resistance and substantially enhance the anticancer action of Doxorubicin and result also suggest that the Doxorubicin delivered by MSNs has minimal premature release in the extracellular environment, which can greatly eliminate side effects of Doxorubicin (58).

\section{Solid lipid nanoparticles (SLN)}

SLN are colloidal drug carrier systems and are similar to nanoemulsions. However, the liquid lipid used in nanoemulsions is replaced by a solid lipid in SLN. SLN can be administered through parenteral (intravenously, intramuscularly or subcutaneously), oral, Rectal, ophthalmic and topical (59). Many studies have been carried on SLN which proves to provide controlled drug delivery, enhancement of bioavailability of entrapped drugs, improvement of tissue distribution and targeting of drugs. To be specific, Keon Wook Kang et al. studied doxorubicinloaded solid lipid nanoparticles on MCF-7/ADR cells (doxorubicin-resistant breast cancer cell line) and result showed that Doxorubicin-loaded solid lipid nanoparticles efficiently enhanced apoptotic cell death through the higher accumulation of doxorubicin in MCF-7/ADR cells in comparison with free doxorubicin (60).

\section{Polymeric nanoparticles}

When the free drugs are administered, they enter the cell by diffusion through the plasma membrane and are recognized by the Pgp pumps whereas these drugs are conjugated to macromolecular carriers, the drug in the form of polymeric prodrug is taken up by endocytosis and subsequently efflux pumps are circumvented inturn reducing the multidrug resistance. Doxorubicin, when conjugated with polymeric dextrans of various molecular weights, its cytotoxicity significantly higher than free doxorubicin when studied on human carcinoma KB-3-1 cells and its multidrug-resistant subclone KB-V-1 cells (61). Similarly, Yang Liu et al. demonstrated that paclitaxel nanocrystal formulation using $\mathrm{D}-\alpha$-tocopheryl polyethylene glycol 1000 succinate have significant advantages over Taxol in achieving better therapeutic effect in Taxol-resistant cancer cells both in vitro and in vivo (62).

\section{Poloxamers}

Poloxamers was invented by Irving Schmolka in the year 1973, which belong to polypropylene oxide (PPO) and polyethylene oxide (PEO) triblock copolymers family which are widely used nanoparticulate engineering for drug delivery systems (63). Due to its amphiphilic structure, poloxamers acts as surfactant and can be used to increase the water solubility of hydrophobic, oily substances and increase the miscibility of two substances with different hydrophobicities. Poloxamers is a potent chemosensitizer of multidrug resistant cancers and its activity includes inhibition of Pgp, inhibits complex I and complex IV of the mitochondria respiratory chain, decreases oxygen consumption and causes ATP depletion in multidrug resistant cells $(64,65)$. Yangqing Zhang et al. studied the cytotoxicity of paclitaxel-loaded poly ( $\varepsilon$-caprolactone) (PCL) / Poloxamer 188 nanoparticles on paclitaxel resistant human breast cancer cell line and found PCL/Poloxamer 188 nanoparticles achieved a significantly higher level of cytotoxicity than both of PCL nanoparticle formulation and $\mathrm{Taxol}^{\circledR}$, which indicates Poloxamer 188 nanoparticles could overcome MDR in human breast cancer cells (66).

\section{Magnetic nanoparticles (MNPs)}

Chemotherapeutic agents bound to a magnetic carrier will be injected in the vascular system and under the influence of an external magnetic field this compound will be held in the targeted area and concentrated at the specific site. MNPs have been studied extensively for targeted delivery of pharmaceuticals. Bao-an Chen et al. evaluated the cytotoxity of daunorubicin (DNR)-loaded magnetic nanoparticles of $\mathrm{Fe}_{3} \mathrm{O}_{4}\left(\mathrm{MNPs}-\mathrm{Fe}_{3} \mathrm{O}_{4}\right.$ ) on $\mathrm{K} 562-\mathrm{n} / \mathrm{VCR}$ cells in mice of different groups and found weight was lower in group which received DNR-MNPs- $\mathrm{Fe}_{3} \mathrm{O}_{4}$ than in other groups. Similarly transcriptions of Mdr-1 and Bcl-2 gene were significantly lower in group which received DNR-MNPs- $\mathrm{Fe}_{3} \mathrm{O}_{4}$ than in other groups. However, there was no difference in the expression of P-glycoprotein. Result indicates DNR-loaded $\mathrm{MNPs}-\mathrm{Fe}_{3} \mathrm{O}_{4}$ can overcome MDR in vivo (67).

\section{Nanoparticle drug delivery system to overcome aqueous solubility}

Drug substances are considered highly soluble when the largest dose of drug is soluble in $<250 \mathrm{~mL}$ water throughout the physiological $\mathrm{pH}$ 
range from $1-8$ but most of the anticancer drugs show poor aqueous solubility. Poor aqueous solubility chemotherapeutics both from plant source and synthetic often demonstrate decreased bioavailability, increased chance of food effect, more frequent incomplete release from the dosage form and higher interpatient variability. However, administration of poor water soluble drugs through systemic route requires solvents like Cremophor EL, Tween (polysorbate)-80, etc. which inturn, lead to severe adverse effects, including acute hypersensitivity reactions, fluid retention, and peripheral neuropathy (68-72). There are two basic approaches to overcome the poor water solubility and poor bioavailability problems (a) Increase of saturation solubility (by complex formation) and (b) Increase of dissolution velocity (Dissolution velocity can be increased by increasing the surface area of the drug powder, i.e. nanonisation) (73).

\section{Nanocrystals}

Nanocrystals increases the dissolution velocity by surface area enlargement (size reduction leads to an increased surface area) and increases the saturation solubility (74). Nanocrystal of hydrophobic drugs like paclitaxel and camptothecin shows significant cytotoxicity by inhibiting the tumor growth in human lung cancer and murine breast cancer than its counter free paclitaxel and camptothecin. The nanocrystals encapsulated over $99 \%$ of the drug with a high ratio of drug to excipient. These nanocrystals also showed significant therapeutic effects via oral administration (75).

\section{Albumin based nanoparticles}

Albumin is a natural carrier of endogenous hydrophobic molecules (such as vitamins, hormones, and other water-insoluble plasma substances), that are bound in a reversible noncovalent manner and enhances penetration by albumin receptor-mediated (gp60) endothelial transcytosis. The albumin coating that surrounds the active drug assists in the transport of the nanoparticles to the interior of the tumor cell that preferentially takes in albumin as a nutrient through the gp60 pathway. Albumin that binds to therapeutic peptide or protein covalently or physically enhances the stability and half-life of the drug (76-83).

Franco Dosio et al. studied paclitaxelalbumin conjugate with two different conjugate populations (with 6 or 30 average molecules of drug linked to each albumin molecule) and found the following (a) Conjugates were stable in physiological solution and in serum whereas the presence of proteases or liver extract released the paclitaxel in a linear fashion, (b) Conjugates maintained high cytotoxicity with efficient cell binding and internalization followed by release of the paclitaxel inside three different tumor cell lines, (c) Both paclitaxel-albumin conjugate followed a bicompartmental model but elimination of the conjugate from the plasma was much slower than the free paclitaxel, giving a relevant rise in area under the curve and mean residence time values after IV administration, (d) The conjugate also released of paclitaxel continuously to the plasma over prolonged periods, thus providing a depot effect and (e) The acute toxicity noted with the standard formulation of taxol was strongly reduced in albuminconjugated preparation (84).

\section{Liposomal formulation}

Liposomes are biodegradable, non-toxic vesicles that have been used to deliver both hydrophilic and hydrophobic agents. Liposomal formulations of several active molecules are currently in preclinical and clinical trials with promising results. The key problems in drug therapy are biodistribution throughout the body which can be overcome by using liposomal formulations. Liposomes protect encapsulated molecules from degradation and can passively target tissues (85). Inclusion of paclitaxel in liposomal formulations has proved to be a good approach to eliminating Cremophor EL and ethanol, improving the drug's antitumor efficacy. Allen Zhang J et al. developed a lyophilized liposome-based paclitaxel (LEPETU) with a mean particle size of $150 \mathrm{~nm}$ which increases the solubility $(0.25 \mathrm{mg} / \mathrm{ml})$ without drug precipitation or change in particle size. In vitro drug release study of LEP-ETU in phosphatebuffered saline ( $\mathrm{pH}$ 7.4) showed that less than $6 \%$ of the entrapped paclitaxel was released after 120 hours, which indicates that the drug is highly stable in an entrapped form at physiologic temperature. Stability data indicated that the lyophilized LEP-ETU was physically and chemically stable for at least 12 months at $2-8{ }^{\circ} \mathrm{C}$ and $25^{\circ} \mathrm{C}(86)$.

\section{Polymeric micelles}

Polymeric micelles are nanosized particles that are made up of polymer chains and are usually spontaneously formed by self-assembly in a liquid, generally as a result of hydrophobic or ion pair interactions between polymer segments. BBSKE is an organic selenium compound chemically named $(1, \quad 2$-[bis $(1, \quad 2$ benzisoselenazol-3(2H)-one)] ethane) with significant antitumor activity. However, BBSKE 
is poorly soluble in water $(2.57 \mu \mathrm{g} / \mathrm{mL})$ and its bioavailability by oral administration is also considerably low. Xinru Li et al. prepared a series of monomethoxy poly (ethylene glycol)-poly (lactide) (mPEG-PLA) diblock copolymers and fabricated with mPEG-PLA micelle. BBSKE was efficiently encapsulated into the micelles by the dialysis method, and the solubility of BBSKE in water was increased up to $82 \mu \mathrm{g} / \mathrm{mL}$. BBSKEloaded polymeric micelle showed enhanced antitumor efficacy and reduced toxic effect compared with BBSKE-HP- $\beta$-CD inclusion at the same dose in $\mathrm{H}_{22}$ human liver cancer cell bearing mouse models. These results suggested that mPEG-PLA polymeric micelle nanoparticles had great potential as nanocarriers for effective solubilization of poorly soluble BBSKE and further reducing side effects and toxicities of the drug (87).

\section{Cyclodextrin based nanoparticles}

Cyclodextrins were used to enhance aqueous solubility and chemical stability of drugs in the beginning and these functionalities were related to their ability to form drug-cyclodextrin inclusion complexes. Cyclodextrin have been shown to participate in various types of non-inclusion complexes with organic salts and water-soluble polymers. Cyclodextrin have also been shown to form aggregates, either alone or in combinations with other excipients which can form dispersed drug delivery systems such as micro and nanoparticles (88).

$\mathrm{Yu}$ Liu et al. studied the inclusion complexation behaviour of paclitaxel with a series of oligo (ethylenediamino) bridged bis ( $\beta$ cyclodextrin) possessing bridge chains in different length of $1-4$ to improve the water solubility of paclitaxel and found bis( $\beta$-cyclodextrin) 1 and 2 are able to solubilize paclitaxel to high levels up to 2 and $0.9 \mathrm{mg} / \mathrm{mL}$, respectively. Result also revealed that the cytotoxicity of these complexes assessed using a human erythroleukemia K562 cell line indicates that the antitumor activity of bis( $\beta$-cyclodextrin) 1-paclitaxel complex is better than that of free paclitaxel (89). Similarly, Agüeros $\mathrm{M}$ et al. encapsulated paclitaxel (PTX) as a complex with three different cyclodextrins $\beta$ cyclodextrin (CD), 2-hydroxypropyl- $\beta$ cyclodextrin (HPCD) and 6-monodeoxy-6monoamino- $\beta$-cyclodextrin (NHCD) and studied the oral bioavailability paclitaxel in rats with a single dose of $10 \mathrm{mg}$ paclitaxel per $\mathrm{kg}$ body weight and result showed that the plasma curves were characterised by a plateau of paclitaxel concentration close to the $\mathrm{C}_{\max }$ from $\mathrm{T}_{\max }$ till $24 \mathrm{~h}$ post-administration. For PTX-CD NP and PTX-
HPCD NP, these sustained levels of the anticancer drug were found to be between 27 and 33-fold higher than the reported value of drug activity whereas the relative oral bioavailability of paclitaxel was calculated to be higher than $80 \%$ (90). These fact shows that cyclodextrin based nanoparticles can significantly alter the solubility and bioavailability and can be utilized to deliver cancer therapeutics with low solubility and bioavailability.

\section{Chitosan based nanoparticles}

Biodegradable polymer that has received a good deal of attention towards oral drug and gene delivery systems is chitosan. Chitosan is nontoxic and biodegradable, with an oral LD50 in mice of over $16 \mathrm{~g} / \mathrm{kg}$. Major factors contributing to chitosan widespread evaluation as a component of oral dosage forms are its safety, its ability to extend residence time in the gastrointestinal tract through mucoadhesion, and its ability to improve absorption by increasing cellular permeability. Absorption enhancement of chitosan was found to depend on both molecular weight and degree of deacetylation (68). Can Zhang et al. studied the possible utilization of amphiphilic N-octyl-Ntrimethyl chitosan (OTMCS) derivatives in solublization and controlled release of hydrophobic anticancer drug, 10hydroxycamptothecin (10-HCPT) and the results showed the solubility of 10-HCPT in aqueous fluid was increased about 80,000-fold from $2 \mathrm{ng} / \mathrm{ml}$ in water to $1.9 \mathrm{mg} / \mathrm{ml}$ in OTMCS micellar (degree of octyl and trimethyl substitution is $8 \%$ and $54 \%$, respectively) solution. In addition, OTMCS was able to modulate the in vitro release of 10-HCPT and improve its pharmacokinetic properties and lactone ring stability in vivo. These data suggested the possible utilization of the amphiphilic micellar chitosan derivatives as carriers for hydrophobic drugs for improving their delivery and release properties (91).

\section{CONCLUSION}

Nanoparticulate drug delivery systems are being studied to overcome limitation of conventional oral dosage form in many therapeutic areas particularly in cancer chemotherapy where most of the anticancer drugs have limited aqueous solubility, lack of targeting cancer tissues and multidrug resistance. Nanoparticle drug delivery system such as folate mediated targeting, transferring targeting are the most studied targeting approach in cancer chemotherapy. However, many other targeting approaches are 
under investigation. Major problem in cancer chemotherapy is multidrug resistance which can be effectively circumvented via Mesoporous Silica Nanoparticles, Solid lipid nanoparticles, Polymeric nanoparticles and Magnetic nanoparticles. Poor aqueous solubility and low bioavailability of cancer chemotherapeutic can be effectively overcome by nanocrystals, albumin based nanoparticles, liposomal formulation, Polymeric micelles, cyclodextrin and chitosan based nanoparticles.

\section{REFERENCES}

1. Nandakumar A. National Cancer Registry Programme, Indian Council of Medical Research, Consolidated report of the population based cancer registries, New Delhi, India: 1990-96.

2. Dinshaw KA, Rao DN, Ganesh B. Tata Memorial Hospital Cancer Registry Annual Report, Mumbai, India, 1999.

3. Ahmedin Jemal, Rebecca Siegel, Elizabeth Ward, Yongping Hao, Jiaquan $\mathrm{Xu}$, Michael $\mathrm{J}$ Thun. Cancer Statistics, 2009. CA Cancer J Clin, 2009; 59:225-249.

4. Jemal A, Siegel R, Xu J, Ward E. Cancer statistics, 2010. CA Cancer J Clin, 2010; 60(5):277-300.

5. Li C. Poly (L-glutamic acid) - anticancer drug conjugates. Adv Drug Deliv Rev, 2002; 54: 695713.

6. Rang, H.P, Dale, M.M, Ritter, J.M, Flower R. Rang and Dale's Pharmacology, 6th edition. Edinburgh, UK Churchill Livingstone, 2007.

7. Kwon GS. Polymeric micelles for delivery of poorly water-soluble compounds. Crit Rev Ther Drug Carrier Syst, 2003; 20:357-403.

8. Luo Y, Prestwich GD. Cancer-targeted polymeric drugs. Curr Cancer Drug Targets, 2002; 2:209226.

9. Simon Benita. Microencapsulation Methods and Industrial Applications. $2^{\text {nd }}$ edn. Informa Healthcare, London, pp 625-671, 2006.

10. Stavrovskaya AA. Cellular mechanisms of multidrug resistance of tumor cells. Biochemistry (Mosc), 2000; 65:95-106.

11. Kinam Park. Nanotechnology: What it can do for drug delivery J Control Release, 2007; 120(1-2):13.

12. Svetlana Gelperina. The Potential Advantages of Nanoparticle Drug Delivery Systems in Chemotherapy of Tuberculosis. American Journal of Respiratory and Critical Care Medicine, 2005; 172:1487-1490.

13. Kwangjae Cho, Xu Wang, Shuming Nie, Zhuo (Georgia) Chen, and Dong M. Shin. Therapeutic Nanoparticles for Drug Delivery in Cancer. Clin Cancer Res, 2008; 14(5):1310-1316.

14. Mohanraj VJ, Chen Y. Nanoparticles - A Review. Tropical Journal of Pharmaceutical Research, 2006; 5 (1): 561-573.
15. Xu Wang, Yiqing Wang, Zhuo (Georgia) Chen, Dong M. Shin. Advances of Cancer Therapy by Nanotechnology. Cancer Res Treat, 2009; 41(1):111.

16. Munn LL. Aberrant vascular architecture in tumors and its importance in drug-based therapies. Drug Discov Today, 2003; 8:396.

17. Wang $X$, Yang $L$, Chen ZG, Shin DM. Application of nanotechnology in cancer therapy and imaging. CA Cancer J Clin, 2008; 58:97-110.

18. Dvorak HF, Nagy JA, Dvorak JT, Dvorak AM. Identification and characterization of the blood vessels of solid tumors that are leaky to circulating macromolecules. American Journal of Pathology, 1988; 133:95-109.

19. Hiroya Hashizume, Peter Baluk, Shunichi Morikawa, John W. McLean, Gavin Thurston, Sylvie Roberge, Rakesh K. Jain and Donald M. McDonald. Openings between defective endothelial cells explain tumor vessel leakiness. Am J Pathol, 2000; 156:1363-1380.

20. Jain RK. Delivery of molecular medicine to solid tumors: Lessons from in vivo imaging of gene expression and function. J Control Release, 2001; 74(1-3):7-25.

21. Jain RK. Transport of molecules across tumor vasculature. Cancer Metastasis Rev, 1987; 6:559593.

22. Brannon Peppas L, Blanchette JO. Nanoparticle and targeted systems for cancer therapy. Adv Drug Deliv Rev, 2004; 56:1649-1659.

23. Jaspreet K. Vasir, Maram K. Reddy, Vinod D. Labhasetwar. Nanosystems in Drug Targeting: Opportunities and Challenges. Current Nanoscience, 2005; 1:47-64.

24. Allen TM, Cullis PR. Drug delivery systems: entering the mainstream. Science, 2004; 303:1818-1822.

25. Nathanson SD, Nelson L. Interstitial fluid pressure in breast cancer, benign breast conditions, and breast parenchyma. Ann Surg Oncol, 1994; 1:333.

26. Kalevi Kairemo, Paola Erba, Kim Bergström, Ernest KJ. Pauwels. Nanoparticles in Cancer. Current Radiopharmaceuticals, 2008; 1:30-36.

27. Lavasanifar A, Samuel J, Kwon GS. Poly (ethylene oxide)-block-poly (L-amino acid) micelles for drug delivery. Advanced Drug Delivery Reviews, 2002; 54(2):169-190.

28. Van Vlerken L, Vyas T, Amiji M. Poly (ethylene glycol)-modified Nanocarriers for Tumor-targeted and Intracellular Delivery. Pharmaceutical research, 2007; 24(8):1405-1414.

29. Emily Gullotti, Yoon Yeo. Extracellularly Activated Nanocarriers: A New Paradigm of Tumor Targeted Drug Delivery. Mol. Pharmaceutics, 2009, 6 (4):1041-1051.

30. Lammers T, Hennink WE, Storm G. Tumortargeted nanomedicines: principles and practice. Br J Cancer, 2008; 99(3):392-397.

31. Elnakat H, Ratnam M. Role of folate receptor genes in reproduction and related cancers. Front Biosci, 2006; 11:506-519. 
32. Elwood PC. Molecular cloning and characterization of the human folate-binding protein cDNA from placenta and malignant tissue culture (KB) cells. J Biol Chem, 1989; 264:1489314901.

33. Giuseppe Destito, Robert Yeh, Chris S. Rae, Finn MG, Marianne Manchester. Folic acid-mediated targeting of cowpea mosaic virus particles to tumor cells. Chem Biol, 2007; 14(10):1152-1162.

34. Guangya Xiang, Jun Wu, Yanhui Lu, Zhilan Liu, Robert J. Lee. Synthesis and Evaluation of a Novel Ligand for Folate-mediated Targeting liposomes. Int J Pharm, 2008; 356(1-2):29-36.

35. Zhaowu Zhang, Jing Jia, Youqun Lai, Yanyan Ma, Jian Weng, Liping Sun Conjugating folic acid to gold nanoparticles through glutathione for targeting and detecting cancer cells. Bioorganic \& Medicinal Chemistry, 2010; 18(15):5528-5534.

36. Fabiana Canal, María J. Vicent, Gianfranco Pasut, Oddone Schiavon (2010) Relevance of folic acid/polymer ratio in targeted PEG-epirubicin conjugates. Journal of Controlled Release, 2010; 146 (3):388-399.

37. Zhang C, Zhao L, Dong Y, Zhang X, Lin J, Chen Z. Folate-mediated poly (3-hydroxybutyrate-co-3hydroxyoctanoate) nanoparticles for targeting drug delivery. Eur J Pharm Biopharm, 2010; 76(1):1016.

38. Xiaobin B. Zhao, Robert J. Lee. Tumor-selective targeted delivery of genes and antisense oligodeoxyribonucleotides via the folate receptor. Advanced Drug Delivery Reviews, 2004; 56(8):1193-1204.

39. Milla M Hänninen, Joonas Haapasalo, Hannu Haapasalo, Robert E Fleming, Robert S Britton, Bruce R Bacon, Seppo Parkkila. Expression of iron-related genes in human brain and brain tumors. BMC Neurosci, 2009; 10:36.

40. Jiang Chang, Youssef Jallouli, Maya Kroubi, Xubo Yuan, Wei Feng, Chun-sheng Kang, Pei-yu Pu, Didier Betbeder. Characterization of endocytosis of transferrin-coated PLGA nanoparticles by the blood-brain barrier. International Journal of Pharmaceutics, 2009; 379 (2):285-292.

41. Tracy R. Daniels, Tracie Delgado, Gustavo Helguera, Manuel L. Penichet. The transferrin receptor part II: Targeted delivery of therapeutic agents into cancer cells. Clinical Immunology, 2006; 121(2):159-176.

42. Yu Zheng, Bo Yu, Wanlop Weecharangsan, Longzhu Piao, Michael Darby, Yicheng Mao, Rumiana Koynova, Xiaojuan Yang, Hong Li, Songlin $\mathrm{Xu}$, L. James Lee, Yasuro Sugimoto, Robert W. Brueggemeier, Robert J. Lee. Transferrin-conjugated lipid-coated PLGA nanoparticles for targeted delivery of aromatase inhibitor $7 \alpha$-APTADD to breast cancer cells. International Journal of Pharmaceutics, 2010; 390 (2):234-241.

43. Rohit S. Mulik, Jukka Mönkkönen, Risto O. Juvonen, Kakasaheb. R. Mahadik, Anant R. Paradkar. Transferrin Mediated Solid Lipid Nanoparticles Containing Curcumin: Enhanced In
Vitro Anticancer Activity By Induction of Apoptosis. International Journal of Pharmaceutics, 2010; 398 (1-2):190-203.

44. Stavrovskaya AA. Cellular mechanisms of multidrug resistance of tumor cells. Biochemistry (Mosc), 2000; 65:95-106.

45. Dean M, Hamon Y, Chimini G. The human ATPbinding cassette $(\mathrm{ABC})$ transporter superfamily. J Lipid Res, 2001; 42:1007-1017.

46. Efferth T. The human ATP-binding cassette transporter genes: from the bench to the bedside. Curr Mol Med, 2001;1:45-65.

47. Cole SP, Bhardwaj G, Gerlach JH, Mackie JE, Grant CE, Almquist KC, Stewart AJ, Kurz EU, Duncan AM, Deeley RG. Overexpression of a transporter gene in a multidrug-resistant human lung cancer cell line. Science, 1992; 258:16501654.

48. Maliepaard M, van Gastelen MA, de Jong LA, Pluim D, van Waardenburg RC, RuevekampHelmers MC, Floot BG, Schellens JH. Overexpression of the BCRP/MXR/ABCP gene in a topotecan-selected ovarian tumor cell line. Cancer Res, 1999; 59:4559-4563.

49. Gupta PK. Drug targeting in cancer chemotherapy: a clinical perspective. J Pharm Sci, 1990; 79:949962.

50. Zee-Cheng RKY, Cheng CC. Delivery of anticancer drugs. Methods Find Exp Clin Pharmacol, 1989; 11:439-529.

51. Belhoussine R, Morjani H, Millot JM, Sharonov S, Manfait M. Confocal scanning microspectrofluorometry reveals specific anthracycline accumulation in cytoplasmic organelles of multidrug-resistant cancer cells. J Histochem Cytochem, 1998; 46:1369-1376.

52. Simon SM. Role of organelle $\mathrm{pH}$ in tumor cell biology and drug resistance. Drug Discovery Today, 1999; 4:32-38.

53. Xiang Rong Song, Zheng Cai, Yu Zheng, Gu He, Feng Yu Cui, Dao Qiong Gong, Shi Xiang Hou, Si Jing Xiong, Xiang Jie Lei, Yu Quan Wei. Reversion of multidrug resistance by coencapsulation of vincristine and verapamil in PLGA nanoparticles. European Journal of Pharmaceutical Sciences, 2009; 37(3-4):300-305.

54. Yanagisawa, Tsuneo; Shimizu, Toshio; Kuroda, Kazuyuki; Kato, Chuzo. The preparation of alkyltrimethylammonium-kanemite complexes and their conversion to microporous materials. Bulletin of the Chemical Society of Japan, 1990; 63:988.

55. Beck JS, Vartuli JC, Roth WJ, Leonowicz ME, Kresge CT. A New Family of Mesoporous Molecular Sieves Prepared with Liquid Crystal Templates. American Chemical Society, 1992; 114 (27):10834-10843.

56. Brian G. Trewyn, Igor I. Slowing, Supratim Giri, Hung-Ting Chen, Victor SY. Lin. Synthesis and Functionalization of a Mesoporous Silica Nanoparticle Based on the Sol-Gel Process and Applications in Controlled Release. Accounts of Chemical Research, 2007; 40(40):846-853. 
57. Dongyuan Zhao, Jianglin Feng, Qisheng Huo, Nicholas Melosh, Glenn H. Fredrickson, Bradley F. Chmelka, Galen D. Stucky. Triblock Copolymer Syntheses of Mesoporous Silica with Periodic 50 to 300 Angstrom Pores. Science, 1998; 279(5350):548-552.

58. Alex M. Chen' Min Zhang, Dongguang Wei, Dirk Stueber, Oleh Taratula, Tamara Minko, Huixin He. Co-delivery of Doxorubicin and Bcl-2 siRNA by Mesoporous Silica Nanoparticles Enhances the Efficacy of Chemotherapy in Multidrug Resistant Cancer Cells. Small, 2009; 5(23):2673-2677.

59. Melike Üner and Gülgün Yener. Importance of solid lipid nanoparticles (SLN) in various administration routes and future perspectives. Int $\mathrm{J}$ Nanomedicine, 2007; 2(3):289-300.

60. Keon Wook Kang, Myung-Kwan Chun, Ok Kim, Robhash Kusam Subedi, Sang-Gun Ahn, JungHoon Yoon, Hoo-Kyun Choi. Doxorubicin-loaded solid lipid nanoparticles to overcome multidrug resistance in cancer therapy. Nanomedicine Nanotechnology Biology and Medicine, 2010; 6(2):210-213.

61. Lam W, Leung $\mathrm{CH}$, Chan $\mathrm{HL}$, and Fong WF Toxicity and DNA binding of dextran-doxorubicin conjugates in multidrug-resistant $\mathrm{KB}-\mathrm{V} 1$ cells: optimization of dextran size. Anticancer Drugs, 2000; 11: 377-384.

62. Yang Liu, Leaf Huang and Feng Liu. Paclitaxel Nanocrystals for Overcoming Multidrug Resistance in Cancer Mol Pharmaceutics, 2010; 7(3):863-869.

63. Santander-Ortega MJ, Jódar-Reyes AB, Csabac N, Bastos-González D, Ortega-Vinuesa JL. Colloidal stability of Pluronic F68-coated PLGA nanoparticles: A variety of stabilisation mechanisms. Journal of Colloid and Interface Science, 2006; 302:522-529.

64. Amit K. Sharma, Li Zhang, Shu Li, David L. Kelly, Valery Yu. Alakhov, Elena V. Batrakova, Alexander V. Kabanov. Prevention of MDR development in leukemia cells by micelle-forming polymeric surfactant. Journal of Controlled Release, 2008; 131 (3):220-227.

65. Daria Yu. Alakhova, Nataliya Y. Rapoport, Elena V. Batrakova, Alexander A. Timoshin, Shu Li, David Nicholls, Valery Yu. Alakhov, Alexander V. Kabanov. Differential metabolic responses to pluronic in MDR and non-MDR cells: A novel pathway for chemosensitization of drug resistant cancers. Journal of Controlled Release, 2010; 142(1):89-100.

66. Yangqing Zhang, Lina Tang, Leilei Sun, Junbo Bao, Cunxian Song, Laiqiang Huang, Kexin Liu, Yan Tian, Ge Tian, Zhen Li, Hongfan Sun, Lin Mei. A novel paclitaxel-loaded poly ( $\varepsilon$ caprolactone)/Poloxamer 188 blend nanoparticle overcoming multidrug resistance for cancer treatment. Acta Biomaterialia, 2010; 6(6):20452052.

67. Bao-an Chen, Bin-bin Lai, Jian Cheng, Guo-hua Xia, Feng Gao, Wen-lin Xu, Jia-hua Ding, Chong Gao, Xin-chen Sun, Cui-rong Xu, Wen-ji Chen,
Ning-na Chen, Li-jie Liu, Xiao-mao Li, Xue-mei Wang. Daunorubicin-loaded magnetic nanoparticles of $\mathrm{Fe} 3 \mathrm{O} 4$ overcome multidrug resistance and induce apoptosis of K562-n/VCR cells in vivo. International journal of nanomedicine, 2009; 4:201-208.

68. Katherine Bowman and Kam W Leong. Chitosan nanoparticles for oral drug and gene delivery. Int $\mathrm{J}$ Nanomedicine, 2006; 1(2):117-128.

69. Heath JR, Davis ME. Nanotechnology and cancer. Annu Rev Med, 2008; 59:251-265.

70. Langer R. Drug delivery and targeting. Nature, 1998; 392:5-10.

71. Shepherd GM. Hypersensitivity reactions to chemotherapeutic drugs. Clin Rev Allergy Immunol, 2003; 24:253-262.

72. Ten Tije AJ, Verweij J, Loos WJ, Sparreboom A. Pharmacological effects of formulation vehicles: implications for cancer chemotherapy. Clin Pharmacokinet, 2003; 42:665-685.

73. Bushrab NF, Müller RH. Nanocrystals of poorly soluble drugs for oral administration. J New Drugs, 2003; 5:20-22.

74. Jens-Uwe A H Junghanns Rainer H Müller. Nanocrystal technology, drug delivery and clinical applications. International Journal of Nanomedicine, 2008; 3(3):295-309.

75. Feng Liu, Ji-Young Park, Yong Zhang, Christine Conwell, Yang Liu, Surendar Reddy Bathula, Leaf Huang. Targeted cancer therapy with novel high drug-loading nanocrystals. Journal of Pharmaceutical Sciences, 2010; 99(8):3542-3551.

76. Henderson IC, Bhatia V. Nab-paclitaxel for breast cancer: a new formulation with an improved safety profile and greater efficacy. Expert Rev Anticancer Ther, 2007; 7(7):919-943.

77. Hawkins MJ, Soon-Shiong P, Desai N. Protein nanoparticles as drug carriers in clinical medicine. Adv Drug Deliv Rev, 2008; 60:876-885.

78. Purcell M, Neault JF, Tajmir-Riahi HA. Interaction of Taxol with human serum albumin. Biochim Biophys Acta, 2000; 1478:61-68.

79. Paal K, Muller J, Hegedus L. High affinity binding of paclitaxel to human serum albumin. Eur J Biochem, 2001; 268:2187-2191.

80. Evelina Miele, Gian Paolo Spinelli, Ermanno Miele, Federica Tomao, Silverio Tomao. Albumin-bound formulation of paclitaxel (Abraxane ${ }^{\circledR}$ ABI-007) in the treatment of breast cancer. International Journal of Nanomedicine, 2009; 4:99-105.

81. Desai N, Trieu V, Yao Z, Louie L, Ci S, Yang A, Tao C, De T, Beals B, Dykes D, Noker P, Yao R, Labao E, Hawkins M, Soon-Shiong P. Increased antitumor activity, intratumor paclitaxel concentrations, and endothelial cell transport of Cremophor-free, albumin-bound paclitaxel, ABI007, compared with Cremophor-based paclitaxel. Clin Cancer Res, 2006; 12(4):1317-1324.

82. MaryAnn Foote. Using nanotechnology to improve the characteristics of antineoplastic drugs: Improved characteristics of nab-paclitaxel 
compared with solvent-based paclitaxel. Biotechnology Annual Review, 2007; 13:345-357.

83. Felix Kratz. Albumin as a drug carrier: Design of prodrugs, drug conjugates and nanoparticles. Journal of Controlled Release, 2008; 132(3):171183.

84. Franco Dosio, Paola Brusa, Paola Crosasso, Silvia Arpicco, Luigi Cattel. Preparation, characterization and properties in vitro and in vivo of a paclitaxel-albumin conjugate. Journal of Controlled Release, 1997; 47(3):293-304.

85. Maria Laura Immordino, Franco Dosio, and Luigi Cattel, Stealth liposomes: review of the basic science, rationale, and clinical applications, existing and potential Int J Nanomedicine, 2006; 1(3):297-315.

86. Allen Zhang J, Gopal Anyarambhatla, Lan Ma, Sydney Ugwu, Tong Xuan, Tommaso Sardone, Imran Ahmad, Development and characterization of a novel Cremophor ${ }^{\circledR}$ EL free liposome-based paclitaxel (LEP-ETU) formulation. European Journal of Pharmaceutics and Biopharmaceutics, 2005; 59(1):177-187.

87. Xinru Li, Zhuoli Yang, Kewei Yang, Yanxia Zhou, Xingwei Chen, Yanhui Zhang, Fei Wang, Yan Liu, and Lijun Ren. Self-Assembled Polymeric
Micellar Nanoparticles as Nanocarriers for Poorly Soluble Anticancer Drug Ethaselen. Nanoscale Res Let, 2009; 4(12):1502-1511.

88. Thorsteinn Loftsson, Dominique Duchene. Cyclodextrins and their pharmaceutical application. International Journal of Pharmaceutics, 2007; 329:1-11.

89. Yu Liu, Guo-Song Chen, Yong Chen, Dong-Xu Cao, Zhi-Qiang Ge, Ying-Jin Yuan. Inclusion complexes of paclitaxel and oligo (ethylenediamino) bridged bis( $\beta$-cyclodextrin)s: solubilization and antitumor activity. Bioorganic \& Medicinal Chemistry, 2004; 12 (22):5767-5775.

90. Agüeros M, Zabaleta V, Espuelas S, Campanero MA, Irache JM. Increased oral bioavailability of paclitaxel by its encapsulation through complex formation with cyclodextrins in poly (anhydride) nanoparticles. Journal of Controlled Release, 2010; 145(1):2-8.

91. Can Zhang, Ya Ding, Liangli (Lucy) Yu, Qineng Ping. Polymeric micelle systems of hydroxycamptothecin based on amphiphilic Nalkyl-N-trimethyl chitosan derivatives. Colloids and Surfaces B: Biointerfaces, 2007; 55(2):192199. 